\title{
Galvanostatic Stripping Chronopotentiometric Study for Determination of Selenium: Pharmacokinetic Application in Experimental Mice
}

\author{
Alexandra Plankova ${ }^{1}$, Kamal M. Matar $^{2, *}$, Peter Rauko ${ }^{3}$, Ladislav Novotny ${ }^{4}$ \\ ${ }^{1}$ Department of Analytical Chemistry, Faculty of Pharmacy, Comenius University, Odbojarov 10, SK-832 32 Bratislava, \\ Slovak Republic \\ ${ }^{2}$ Department of Applied Therapeutics, Faculty of Pharmacy, Kuwait University, PO Box 24923 Safat, 13110 Kuwait \\ ${ }^{3}$ Cancer Research Institute, Slovak Academy of Sciences, Vlarska 7, SK-833 91 Bratislava, Slovak Republic \\ ${ }^{4}$ Department of Pharmaceutical Chemistry, Faculty of Pharmacy, Kuwait University, PO Box 24923 Safat, 13110 Kuwait
}

Received, August 11, 2009; Revised, August 9, 2010; Accepted, September 22, 2010; Published, September 24, 2010

\begin{abstract}
Purpose: The aim of this study was to determine the selenium content in various tissues of the mouse employing the galvanostatic stripping chronopotentiometry (SCP) technique and to investigate the distribution profile of selenium as well as its pharmacokinetics in a mouse model. Methods. The animals received $0.25 \mu \mathrm{g} / \mathrm{g}$ Se orally for 5 days. Samples of whole blood and various tissues comprising kidney, liver and brain were harvested from mice and then analysed for Se content employing the SCP technique. Results. The SCP method was validated over Se concentration range of $10-100 \mathrm{ng} / \mathrm{mL}$ and showed good linearity $\left(\mathrm{r}^{2}>\right.$ 0.999). The precision (over 5 days) of the assay in various mouse tissues (liver, kidney, and brain) ranged from 0.03 to $2.9 \%$ with accuracy results that varied from -6.69 to $0.28 \%$. The mean $(n=5)$ recoveries of Se from the mouse tissues ranged from 93.31 to $100.28 \%$. The lower limit of Se detection in the mouse tissues was 0.2 $\mathrm{ng} / \mathrm{mL}$. The present method was successfully applied in evaluating the distribution of Se in various tissues as well as its pharmacokinetics in the mouse model. The Se tissue concentrations in the mouse model showed that the maximum Se levels in most tissues were attained within 3-4 days following its administration. Furthermore, the pharmacokinetic profile of Se in the mouse model indicates that the element is slowly absorbed from the gastrointestinal tract (GIT) reaching a plateau in 4 days and then it is slowly eliminated from the body with a half-life of about 4.5 days. Conclusions. The present SCP method was employed to analyse Se in various mouse tissues. The method was characterized by excellent performance parameters necessary for the determination of Se in biological matrices. Se distributes in whole blood as well as into various tissues of the mouse with high concentrations in the kidney and liver and low levels in the blood and brain tissues. The absorption of Se from the GIT was very slow and the data suggest that the elimination of Se seems to be through the kidney at a very slow rate as well. The data of the present study thus suggest that Se remains in the mouse body for a long period of time.
\end{abstract}

\section{INTRODUCTION}

Selenium, as an electronegative non-metal element, is usually present in nature as toxic selenite ion or inorganic selenides or selenates. It is also present in enzymes of living organisms (1). Selenium functions in the body as an important dietary antioxidant and is now widely recognized as an essential and important component of the active sites of a number of enzymes $(2,3)$. It is an essential trace element that is required for the formation of several selenocysteine-containing selenoproteins with key roles in important metabolic processes (4). Its physiologically optimal daily requirement was found to be around $40 \mu \mathrm{g}$ in humans (5). Se intake exceeding this amount may lead to selenosis that may result in various problems including gastrointestinal disorders, neurological damage and other complications (6). On the other hand, the minimum daily Se required for prevention of Keshan disease and clinically relevant signs of dietary inadequacy as recommended by WHO is 19 $\mu \mathrm{g}$ (7). It is known that dietary Se deficiency has been associated with a number of chronic diseases such as Keshan disease, cardiovascular diseases, cancer, asthma, arthritis and many others $(3,8)$. The exact mechanism of Se in the biological system is not yet fully understood.

Corresponding Author: Dr. Kamal M. Matar, Dept. of Applied Therapeutics, Faculty of Pharmacy, Kuwait University, Safat 13110, Kuwait. E-mail: kamal@hsc.edu.kw 
However, it has been proposed that Se competes with sulfur for sites at which sulfur normally plays a role in cellular metabolism (9).

Because of the biological importance of $\mathrm{Se}$ there exists a need for reliable methods to determine Se in biological samples as well as in tissues. This will help to clarify various biochemical mechanisms in which Se is involved.

There are few analytical methods for the determination of Se which have been reported in the literature. These include methods such as radioisotope-induced X-ray fluorescence (10), high performance liquid chromatography-hydride generation-(fast sequential) atomic absorption spectrometry (11), capillary electrophoresis on-line coupled with hydride generation-atomic fluorescence spectrometry (12), and especially high-performance liquid chromatography coupled with inductively coupled plasma mass spectrometry $(13-15)$ which is appropriate for the determination of inorganic substances. Moreover, electrochemical methods such as stripping chronopotentiometry (16 -19) or linear sweep and square-wave voltammetry (20), have also been used for the determination of Se.

Methods involving electrochemical stripping techniques have been shown to be the most sensitive and reliable for electroactive elements such as $\mathrm{Zn}, \mathrm{Pb}, \mathrm{Cd}$, Se and others (21). With these methods however, the deposit is stripped either electrochemically, by applying a constant dissolution current (galvanostatic or constant current chronopotentiometry), or chemically using an oxidant (potentiometric stripping analysis). In both situations, the change of the potential of the working electrode is recorded and assessed. The main methods used for the determination of low concentrations of elements in biological matrices are differential pulse polarography, anodic and cathodic stripping voltammetry, adsorptive stripping voltammetry and galvanostatic stripping chronopotentiometry.

The galvanostatic stripping chronopotentiometry (SCP) technique is used mainly for the quantitative determination of low concentrations of heavy metals such as lead, cadmium, copper, zinc and others in various types of samples (21). Occasionally, terms such as inverse chronopotentiometry, coulometric stripping potentiometry or galvanic/galvanostatic stripping chornopotentiometry have been used for this electrochemical method.

An advantage of the SCP method is that no current is present at the working electrode during the stripping phase of the analysis. Consequently, the use of this method is not affected by interferences of electroactive particles present in the analytical system. Furthermore, the potential of a working electrode does not change linearly but it stays almost unchanged during oxidation of an individual metal until the metal is fully dissolved. The apparent advantage of the SCP technique is that the time instead of current is measured during the analysis. Furthermore, potentiometric analyzers are simpler compared to polarographic analyzers as they do not contain various components necessary for changing potential during metal dissolution. The SCP limits of determination are in the range of $1.0 \times 10^{-7}-1.0 \times 10^{-9}$ mol. $1^{-1}$, which makes it possible for the simultaneous determination of several metals without any additional pre-separation step and in addition, the analysis itself is not timeconsuming (21). The SCP method is based on a two-step electrochemical method of analysis. The first step for the determination of Se involves preconcentration of Se that is deposited on a working electrode with a constant potential. The second step (stripping) involves oxidation of the deposited Se by a chemical oxidant at a constant current. The analytical determination that reflects the potential of the working electrode as a function of time is then used to determine Se in samples. The stripping time itself serves as a parameter for the determination of non-metal quantity. Before analysis by electrochemical analytical methods, it is necessary to mineralize the samples containing inorganic as well as organic compounds. The aim of mineralization however, is for complete destruction of electrochemically active organic substances and groups that can interfere with the determination of small or trace quantities of mineral compounds (22).

The aim of the present study was to employ the $\mathrm{SCP}$ technique to investigate Se distribution in mice and to obtain information on Se pharmacokinetics since the literature lacks novel information in this regard (9) because the assay methods used previously were not as reliable and accurate as the method used in the present study. 


\section{MATERIALS AND METHODS}

\section{Chemicals}

The following sources of $\mathrm{Se}$ were used: Selen and Selezin ACE were obtained from Walmark (Trinec, Czech Republic), Zinkosel was obtained from ACHAT Pharma (Praha, Czech Republic) and Selen also obtained from Naturica (Myjava, Slovak Republic). These preparations contained other additional nutraceuticals such as zinc (Selezin ACE, Zinkosel), minerals, vitamins A, C and E. Moreover, Selen (Naturica) contained selenium with green tea and vitamin E. All other chemicals used were of the highest purity available and are commercially available.

\section{Selenium Administration}

All Se tablets were of commercial origin with various nominal Se content (Table 1). Suspensions in 20\% Tween-60 (Fluka, Germany) were prepared from selenium-containing tablets. Briefly, tablets were pulverized and then the powder containing equivalent Se dose was weighed. A Tween-60: water (1:4) mixture was used to prepare the suspension. A $100 \mu \mathrm{L}$ of Tween-60 was added to pulverized Se and then $400 \mu \mathrm{L}$ of water was added to this mixture. The prepared suspension was then administered orally to the mouse using a feeding tube in a volume equivalent to a $0.25 \mu \mathrm{g}$ of Se per $\mathrm{g}$ of body weight of experimental mouse per day at days $0,1,2,3$ and 4 of the experiment. Selenium was in an inorganic form of sodium selenite.

\section{Experimental Animals}

Inbred DBA/2J mice of both sexes were obtained from the breeding facility of the Cancer Research Institute of SAS (Bratislava, Slovak Republic). The workstation is certified for performing scientific research on animals. The Ethics Committee of the Cancer Research Institute approved the experiments of this work. The in vivo experiments were performed with full adherence to the European Community Guidelines Principles for Care and Use of Laboratory Animals (23).

A total number of 108 mice were used in this study. Of these, 16 animals were used as controls and 92 as the treated group. The treatment group was randomly assigned and divided into 4 subgroups. The control group $(\mathrm{n}=16)$ was subdivided into 4 subgroups, each containing 4 mice. Twelve of the control animals were anesthetized and then liver, kidney and brain tissues were harvested and weighed ( $\mathrm{n}=4$ for each tissue). The remaining 4 mice of the control group were also anesthetized and then blood samples were withdrawn from the heart and transferred into preheparinized test tubes.

\begin{tabular}{|c|c|c|}
\hline $\begin{array}{l}\text { Se product } \\
\text { (Producer) }\end{array}$ & Tablet weight (g) & $\begin{array}{c}\text { Nominal Se } \\
\text { content } \\
\text { ( } \mu \text { g/tablet) }\end{array}$ \\
\hline Selen (Walmark) & 0.33 & 50.00 \\
\hline Selezin (ACE) & 1.00 & 100.00 \\
\hline Selen (Naturica) & 0.25 & 20.00 \\
\hline Zinkosel (ACHAT) & 0.62 & 40.00 \\
\hline
\end{tabular}

For the treatment group ( $\mathrm{n}=92)$, commercial Se products (Table 1) were orally administered to the mice in the 4 subgroups at a dose of $0.25 \mu \mathrm{g} \mathrm{Se} / \mathrm{g}$ body weight at days 0, 1, 2, 3 and 4. Each commercial Se product was administered to one mouse in each subgroup at each time point, i.e., the four different Se products were administered to 4 animals at each time point. Tissue harvesting and blood sample collections were performed 24 hours after the last Se administration. This means that the first samples were collected $24 \mathrm{~h}$ following the first Se administration (at day 0) and the second samples $24 \mathrm{~h}$ following the second Se dose administration (after days 0 and 1). Se administration continued until the last dose which was on day 4 and the samples were collected on day 5 ( $24 \mathrm{~h}$ later). However, kidney, liver and brain were harvested 4 days later following the last Se administration (i.e., on day 8). Following tissue harvesting and blood collection, the animals were immediately sacrificed by applying deep anesthesia. All tissues harvested and blood samples collected were immediately frozen at $-20{ }^{\circ} \mathrm{C}$ pending analysis.

\section{Mineralization of Biological Samples}

Mineralization of tissue samples and blood harvested from experimental animals was performed at high pressure in an autoclave (Laboratorni autoklav ZA-1.JZD 'Pokrok' Zahnasovice, Kromeriz, Czech Republic) by a wet method. In the mineralization process, it was necessary to monitor the effect of temperature, pressure, type and volume of mineralization agent on efficiency of mineralization. Following 
experimental results, the optimal conditions for mineralization of analyzed samples were determined to be as follows: optimal temperature of $160{ }^{\circ} \mathrm{C}$ in $120 \mathrm{~min}$, at high pressure (autoclaveconvenience heating) using $1.5 \mathrm{~mL}$ of concentrated $\mathrm{HNO}_{3}$ as the mineralization agent. The convenience heating was performed in the autoclave in order to set the temperature for mineralization. Because the autoclave is heated up by a flow of hot air, it warms uniformly and mineralization (decomposition) of a biological sample becomes uniform as well. In this method, nitric acid was used as a mineralizing agent and hence a solution was formed (as required for the present assay method) following the mineralization process. The solution produced was then dried using high temperatures $\left(160{ }^{\circ} \mathrm{C}\right.$; hot air dryer).

\section{Galvanostatic Stripping Chronopotentiometry Analysis}

The content of Se was analysed in samples with the SCP method using an electrochemical analyser (ECA-Sensor, Istran sro, Bratislava, Slovak Republic). The following system of electrodes was used to determine the content of Se: reference electrode-silver chloride electrode $(\mathrm{Ag} / \mathrm{AgCl})$; working electrode-replaceable gold-coated carbon electrode. Maximum potential of the dissolution peak (Ep) of Se in basic electrolyte consisting of sulphuric and hydrochloric acids $\left(\mathrm{C}_{\mathrm{H} 2 \mathrm{SO} 4}=1.0 \times 10^{-1}\right.$ mol. $1^{-1} ; \mathrm{C}_{\mathrm{HCl}}=1.0 \times 10^{-3}$ mol. $\left.1^{-1}\right)$ measured against silver chloride electrode was $870 \mathrm{mV}$. Ep value of zinc in an acetate buffer $(\mathrm{pH}=4.4)$ was $-825 \mathrm{mV}$.

The sample preparation procedure was optimized with respect to the optimal mineralization conditions. The product of mineralization (residue) was obtained from the solution formed as a result of the treatment of the biological sample (tissue or blood) with the concentrated nitric acid. The formed solution however, was quantitatively transferred to a $25 \mathrm{~mL}$ measuring flask, the electrolyte $\left(\mathrm{H}_{2} \mathrm{SO}_{4}\right.$ and $\mathrm{HCl}$ ) was added and the mixture was adjusted to the required volume of the measuring flask using de-ionized water. A $25 \mathrm{~mL}$ of a biological sample together with the electrolyte was placed in a dry quartz beaker. The sensor was placed into this solution and the background signal as well as the sample signal were measured. Following this step, a standard was added to the measured solution and then the signal measurement was performed. The background signal was then automatically subtracted from the signals of the sample and that of the sample together with the standard.

The results obtained were verified using various standard solutions prepared from certified reference materials provided by the Slovak Metrology Institute, Bratislava. Metrology parameters for SCP analysis tolerate 5\% variation for Se determination in the range of $10-100$ $\mathrm{ng} / \mathrm{mL}$. The operating conditions for $\mathrm{Se}$ determination by the SCP method were the same as previously reported (21).

\section{Validation of the SCP Method}

The SCP profile of Se obtained in the working background electrolyte solution was characterized by a single peak with a maximum potential of 870 $\mathrm{mV}$. For the quantitative determination of $\mathrm{Se}$ content, the area of the Se peak was evaluated. Quantitative analysis of Se was employed using the multiple point standard addition method (24). The standard addition method was performed using a certified Se reference standard of $1.0 \mathrm{mg} / \mathrm{mL}$. Calibration standards were prepared employing standard dilutions of the Se reference solution (1.0 $\mathrm{mg} / \mathrm{mL}$ ) using $2 \% \mathrm{HNO}_{3}$ as supporting electrolyte. The calibration standards were prepared at concentrations of $10,30,50,80$, and $100 \mathrm{ng} / \mathrm{mL}$ and analysed in replicates of five. The slope, intercept, and coefficient of determination $\left(\mathrm{r}^{2}\right)$ were determined by a least squares linear regression model. The back calculated Se concentrations as well as various parameters of the regression equation were automatically calculated employing the Ecapol program, version 2.1 (Istran sro, Bratislava, Slovak Republic). The selected calibration range was appropriate for application of the SCP method for determination of Se in mouse tissues. Precision and accuracy of the present assay method were assessed by spiking aliquots of liver, kidney, and brain tissues with $\mathrm{Se}$ at 4 different concentrations and then extracted in replicates of 5 over the period of 5 days using the present method. The \%RSD, serves as a measure of precision (reproducibility) and \% deviation from the nominal concentration (bias, \%) serves as a measure of accuracy. On the other hand, the precision and accuracy in mice blood were not assessed using the present method because of small sample volumes obtained which were not appropriate for the present method. However, these parameters were evaluated by comparison of the Se content in human plasma 
obtained by the SCP method with those obtained using a comparative analytical method- atomic absorption spectrometry with hydride generation (HGAAS) as previously reported (25). The recovery was assessed by spiking the liver, kidney and brain tissues with $\mathrm{Se}$ at four different concentrations. The spiked samples were then extracted and analysed using the present SCP method. Recovery was determined by comparing the Se content found after extraction to that before extraction. Detection limit (LOD) was obtained by $L O D=3 S_{b} / b$, where $S_{b}$ was the estimated standard deviation of the blank and $b$ was the sensitivity of the measurement which was represented by: $b=$ $R z F V /(i \mathrm{Mr})$; where $R$ is the electrochemical extraction, $z$ the charge number, $F$ the Faraday constant $(96484 \pm 0.27 \mathrm{C} / \mathrm{mol}), V$ the sample content, $i$ the dissolving current, and $\mathrm{Mr}$ the gram atomic weight (26).

\section{Pharmacokinetic Analysis}

The pharmacokinetic parameters for Se in mouse blood were estimated by standard noncompartmental methods. The maximum Se blood concentration $\left(C_{\max }\right)$ and the time needed to attain this concentration $\left(T_{\max }\right)$ were directly obtained from the Se blood profiles; the Se blood elimination half-life $\left(t_{1 / 2}\right)$ values were calculated from $L n 2 / k_{e l}$. The area under the Se blood concentration-time curve $\left(A U C_{0-t}\right)$ was calculated from the measured data points from time zero to time of last quantifiable concentration by the linear trapezoidal rule and the area under the Se blood concentrationtime curve extrapolated to infinity $\left(A U C_{0-\infty}\right)$ was calculated using the equation: $A U C_{0-\infty}=A U C_{0-t}+$ $\mathrm{C}^{*} / \mathrm{k}_{\mathrm{el}}$, where $\mathrm{C}^{*}$ is the last quantifiable Se blood concentration. The Se clearance $(C l / F)$ was calculated as the ratio of Dose/AUC $0_{-\infty}$. The pharmacokinetic calculations were performed using WinNonLin software (version 5.1, Pharsight Co., CA, USA).

\section{RESULTS AND DISCUSSION}

Selection of an appropriate method for qualitative and quantitative determinations of various elements such as $\mathrm{Zn}, \mathrm{Pb}, \mathrm{Cd}$ and $\mathrm{Se}$ in samples of various sources depends on goals of the analysis, characteristics of the sample, amount of the element in the sample as well as the availability of reliable instrumentation. Selection of a reliable method however, is significantly influenced by its specificity, sensitivity and quantification limit.

In the present work however, the selection of a suitable method of destruction of a biological material was necessary, i.e for the determination of low Se concentrations, organic constituents of biological samples were decomposed by the wet procedure using nitric acid.The SCP method performance and validation are shown in Tables $2 \& 3$. The calibration curves $(n=5)$ showed good linearity over the Se concentration range of $10-$ $100 \mathrm{ng} / \mathrm{mL}$ (Table 2). The data on precision and accuracy of the present assay are demonstrated in Table 3. The precision (over 5 days) of the present assay, presented as RSD, \%, in various mouse tissues (liver, kidney and brain) ranged from 0.03 to $2.90 \%$. The accuracy, presented as percentage bias against nominal Se concentration, ranged from 6.69 to $0.28 \%$. The results of the present method demonstrated adequate accuracy, precision and reproducibility. The mean $(n=5)$ recovery of Se from various mouse tissues (liver, kidney, and brain) ranged from 93.31 to $100.28 \%$, (Table 3). The limit of Se detection using the present SCP method was $0.2 \mathrm{ng} / \mathrm{mL}$. No interferences from iron, chlorides or electroactive organic compounds were found in the SCP profiles when analyzing the standards as well as real (mineralized) samples. These parameters confirmed the suitability of the SCP method for Se determination in tissues and blood samples.

\begin{tabular}{cccc}
\hline \multicolumn{4}{c}{ Table 2. Summary of calibration curves data } \\
\hline Cali \# & Intercept & Slope & $\mathbf{r}^{2}$ \\
\hline 1 & 0.614 & 0.985 & 0.9998 \\
2 & 1.245 & 0.986 & 0.9995 \\
3 & 0.398 & 0.994 & 0.9992 \\
4 & 0.304 & 1.001 & 0.9997 \\
5 & -0.55 & 1.014 & 0.9997 \\
Mean & 0.402 & 0.996 & 0.9996 \\
SD & & 0.012 & 0.0002 \\
RSD, \% & & 1.203 & 0.024 \\
\hline
\end{tabular}

The present SCP method was used for analysis of Se-containing products as presented in Table 4. Furthermore, the SCP method was applied for the determination of Se in various tissues of the mouse. 


\begin{tabular}{|c|c|c|c|c|c|}
\hline Tissue & $\begin{array}{c}\text { Nominal conc. } \\
(\mathrm{ng} / \mathrm{mL})\end{array}$ & $\begin{array}{c}\text { Found conc. }(\mathrm{ng} / \mathrm{mL}) \\
\left({ }^{\mathrm{a}} \text { mean } \pm \mathrm{SD}\right)\end{array}$ & RSD, \% & ${ }^{\mathrm{b}}$ Bias, \% & ${ }^{\circ}$ Recovery,\% \\
\hline \multirow[t]{4}{*}{ Liver } & 14.14 & $14.13 \pm 0.03$ & 0.21 & -0.07 & 99.93 \\
\hline & 29.14 & $27.25 \pm 0.04$ & 0.16 & -6.49 & 93.51 \\
\hline & 59.14 & $58.16 \pm 0.05$ & 0.08 & -1.66 & 98.34 \\
\hline & 89.14 & $88.36 \pm 0.07$ & 0.08 & -0.88 & 99.12 \\
\hline \multirow[t]{4}{*}{ Kidney } & 8.87 & $08.59 \pm 0.04$ & 0.5 & -3.16 & 96.84 \\
\hline & 23.87 & $22.56 \pm 0.07$ & 0.03 & -5.49 & 94.51 \\
\hline & 53.87 & $54.02 \pm 0.03$ & 0.06 & 0.28 & 100.28 \\
\hline & 83.87 & $83.29 \pm 0.07$ & 0.08 & -0.69 & 99.3 \\
\hline \multirow[t]{4}{*}{ Brain } & 1.9 & $1.79 \pm 0.05$ & 2.9 & -5.79 & 94.21 \\
\hline & 16.9 & $15.77 \pm 0.10$ & 0.62 & -6.69 & 93.31 \\
\hline & 46.9 & $45.94 \pm 0.13$ & 0.29 & -2.05 & 97.95 \\
\hline & 76.9 & $75.98 \pm 0.07$ & 0.1 & -1.20 & 98.8 \\
\hline
\end{tabular}

In this regard, various Se products were used (Table 1). The purpose of using different Se products was to investigate whether Se present in different commercial products will have an influence on its tissue distribution profile in the mouse model. Selenium tissue distributions (obtained from four tested Se products) after oral administration (0.25 $\mu \mathrm{g} / \mathrm{g}$ ) in mice (each Se product was tested in four mice) are shown in Fig.1. The results demonstrated lack of significant differences in Se distribution among various Se products. Furthermore, Se tissue distribution in mice was found to be high in the kidney and liver whereas low levels were found in the blood and brain. This finding concurs with an earlier study in humans (9). The highest Se concentration in the kidney suggests that $\mathrm{Se}$ is presumably excreted primarily via the kidney as previously reported (9). The highest Se concentration in the kidney and liver tissues was attained 2 days following its administration whereas in the blood and brain tissues it was attained after 4 days. On the other hand, the decline of $\mathrm{Se}$ concentration is very slow and it occurs in a parallel manner in all studied tissues (Fig. 1). Furthermore, Se blood levels in mice show that the element is slowly absorbed reaching a maximum blood concentration after 3 days and then it is slowly excreted from the body with a mean elimination half-life of 4.5 days and a mean clearance of 2.1 $\mathrm{mL}$ /day (Table 5). The present SCP method was previously successfully employed for the assessment of the relationship between atopic dermatitis and Se concentration in human plasma (16). The findings of that study demonstrated a good correlation between Se plasma concentration and atopic dermatitis in humans. In that study (16) however, the matrix used was human plasma whereas, in the present study the SCP method was used for the determination of Se in mouse blood as well as in various tissues and then further applied to study Se pharmacokinetics and its distribution profile in the mouse model.

\section{CONCLUSIONS}

The present SCP method was characterized by excellent performance parameters. The method was applied in the mouse model to assess Se distribution profile in this model. The study showed that the absorption of Se from the gastrointestinal tract in the mouse was very slow and the tissue distribution was almost into most tissues with the highest concentrations in the kidney and liver whereas low levels of the element were observed in the blood and brain tissues. 


\begin{tabular}{|c|c|c|c|}
\hline Se product (Producer) & $\begin{array}{l}\text { Nominal Se content } \\
(\mu \mathrm{g} / \text { tablet })\end{array}$ & $\begin{array}{l}{ }^{*} \text { Mean }( \pm \text { SD) found Se } \\
\text { content }(\mu \mathrm{g} / \text { tablet })\end{array}$ & RSD., \% \\
\hline Selen (Walmark) & 50.00 & $50.83 \pm 0.46$ & 0.91 \\
\hline Selezin (ACE) & 100.00 & $99.79 \pm 0.48$ & 0.48 \\
\hline Selen (Naturica) & 20.00 & $19.87 \pm 0.51$ & 2.57 \\
\hline Zinkosel (ACHAT) & 40.00 & $40.08 \pm 0.52$ & 1.30 \\
\hline
\end{tabular}

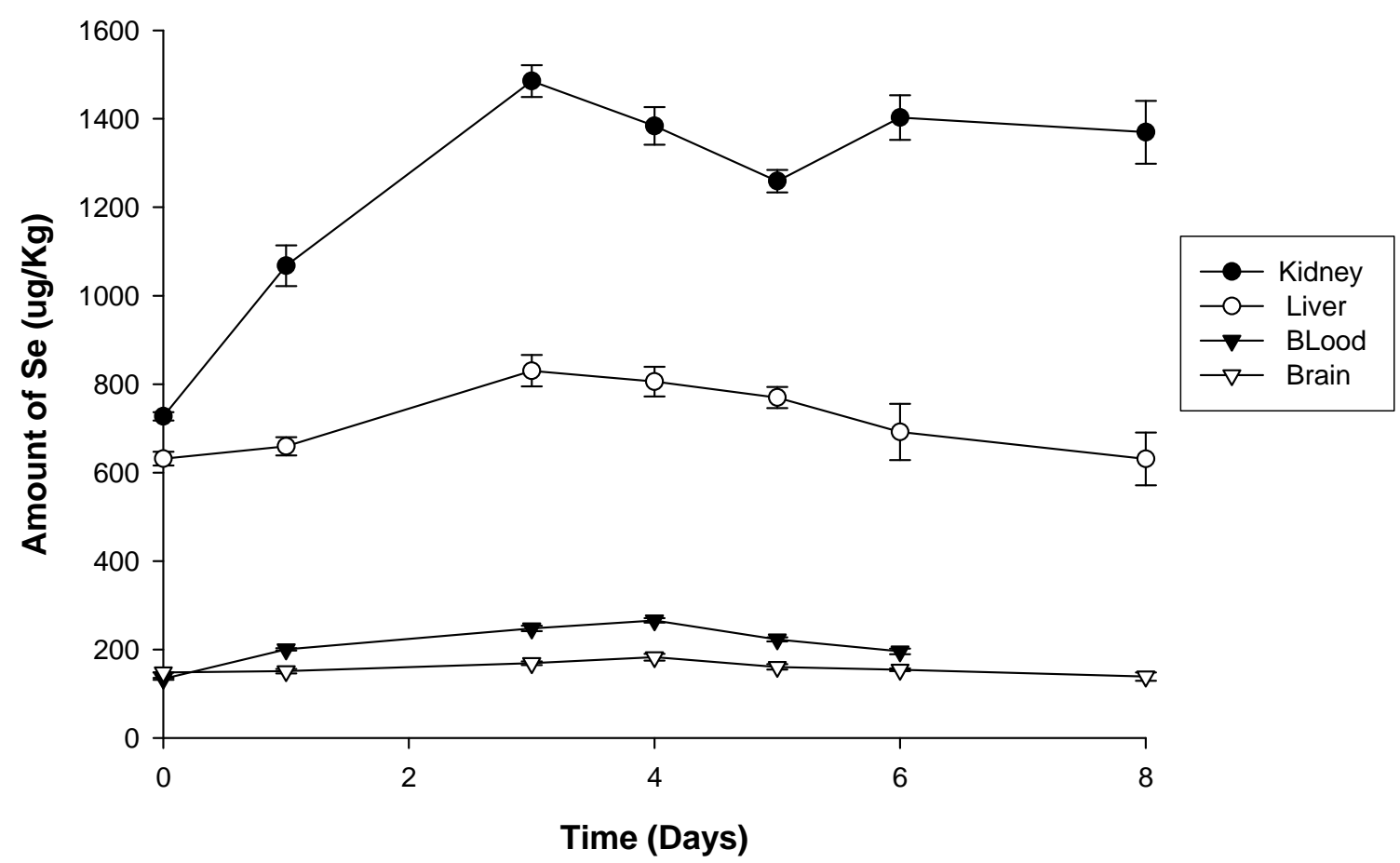

Figure 1. Distribution of selenium in various mouse tissues following an oral Se dose of $0.25 \mu \mathrm{g} / \mathrm{g}(\mathrm{n}=4)$

Table 5. Mean ( \pm S.D.) pharmacokinetic parameters of selenium in mouse blood following daily oral doses (6 days) of selenium $(0.25 \mu \mathrm{g} / \mathrm{g})$ to four mice.

\begin{tabular}{lc}
\hline \multicolumn{1}{c}{ Parameter } & Mean \pm S.D \\
\hline $\mathrm{C}_{\max }$ (ng/ml) & $265.93 \pm 5.09$ \\
$\mathrm{~T}_{\max }$ (day) & $4.00 \pm 0.00$ \\
$\mathrm{t}_{1 / 2}$ (day) & $4.45 \pm 0.21$ \\
$\mathrm{Cl} / \mathrm{F}(\mathrm{ml} /$ day $)$ & $2.08 \pm 0.21$ \\
\hline
\end{tabular}


Our findings demonstrate that Se tissue distribution in the mouse model is very similar to that in humans and therefore the mouse model may be suitable for further tissue distribution studies of Secontaining compounds. Furthermore, the estimated half-life of Se in the mouse was found to be 4.5 days with low clearance values of $2.1 \mathrm{~mL} /$ day. The data of the present study suggest that Se remains in the mouse body for a long period of time. Since Se is a biologically important trace element and is widely recognized as an important dietary antioxidant, the present method however will be of great value in situations where a reliable assay is needed for obtaining accurate results that help in clarifying its mechanism in many oxidative reactions in biological systems.

\section{ACKNOWLEDGEMENTS}

The present work was supported by Slovak Grant Agency VEGA, Grant numbers: 2/7088/27 and 1/4299/07.

\section{REFERENCES}

1. Combs, G.F., Gray, W.P., Chemopreventive agents: selenium. Pharmacol Ther, 79:179 - 192, 1998.

2. Stadtman, T., Selenocysteine. Ann Rev Biochem, 65:83 - 100, 1996.

3. Rayman, M., The importance of selenium to human health. Lancet, 356:233 - 241, 2000.

4. Thomson, C., Assessment of requirements for selenium and adequacy of selenium status: a review. Eur J Clin Nutr, 58:391 - 402, 2004.

5. Duffield, A., Thomson, C., Hill, K., Williams, S., An estimation of selenium requirements for New Zealanders. Am J Clin Nutr, 70:896 - 903, 1999.

6. Wilber, C.G., Toxicology of selenium: a review. Clinical Toxicology, 17:171 - 230, 1980.

7. WHO/FAO/IAEA., Trace elements in human nutrition and health. Geneva: World Health Organization. 1996.

8. Clark, L., Combs, G., Turnbuli, B., et al.,. Effect of selenium supplementation for cancer prevention in patients with carcinoma of the skin. $J$ Am Med Assoc, 276:1957 - 63, 1996.

9. Oehme, F., Mechanisms of heavy metal toxicities. Clin Toxicol, 5:151 - 167, 1972.

10. Tariq, M.A., Preiss, I.L., Determination of selenium in animal tissue samples using radioisotope-induced X-ray fluorescence. $J$ Radioanal Nucl Chem, 163:355 - 362, 1992.

11. Niedzielski, P., The new concept of hyphenated analytical system: simultaneous determination of inorganic arsenic(III), arsenic(V), selenium(IV) and selenium(VI) by high performance liquid chromatography-hydride generation-(fast sequential) atomic absorption spectrometry during single analysis. Anal Chim Acta, 551:199 - 206, 2005.

12. Lu, C.Y., Yan, X.P., Capillary electrophoresis online coupled with hydride generation-atomic fluorescence spectrometry for speciation analysis of selenium. Electrophoresis, 26:155 - 160, 2005.

13. Koyama, H., Omura, K., Ejima, A., Kasanuma, Y., Watanabe, C., Satoh, H., Separation of seleniumcontaining proteins in human and mouse plasma using tandem high-performance liquid chromatography columns coupled with inductively coupled plasma mass spectrometry. Anal Biochem, 267:84 - 91, 1999.

14. Shigeta, K., Matsumura, K., Suzuki, Y., Shinoharo, A., Furuta, N., Distribution and dynamic pathway of selenium species in selenium-deficient mice injected with 82Se-enriched selenite. Anal Sci, 24:1117 $1122,2008$.

15. Dernovics, M., Lobinski, R., Speciation analysis of selenium metabolites in yeast-based food supplements by ICPMS-assisted hydrophilic interaction HPLC-hybrid linear ion trap/orbitrap MSn. Anal Chem, 80:3975 - 3984, 2008.

16. Plankova, A., Mikus, P., Havranek, E., Determination of selenium in clinical plasma samples related to atopic dermatitis study by chronopotentiometric stripping method. Pharmazie, 65:327 - 330, 2010.

17. La Pera, L., Lo Curto, R., Di Bella, G., Dugo, G., Determination of some heavy metals and selenium in Sicilian and Calabrian citrus essential oils using derivative stripping chronopotentiometry. J Agric Food Chem, 53:5084 - 5088, 2005.

18. Riso, R.D., Waeles, M., Garbarino, S., Le Corre, P., Measurement of total selenium and selenium(IV) in seawater by stripping chronopotentiometry. Anal Bioanal Chem, 379:1113 - 1119, 2004.

19. Serrano, N., Diaz-Cruz, J.M., Arino, C., Esteban, M., Stripping chronopotentiometry in environmental analysis. Electroanal, 19:2039 - 2049, 2007.

20. Rahman, M.A., Park, D.S., Won, M.S., Park, S.M., Shim, Y.B., Selective electrochemical analysis of various [heavy] metal ions at an EDTA-bonded conducting polymer-modified electrode. Electroanalysis, 16:1366 - 1370, 2004.

21. Stroffekova, O., Plankova, A., Janosova, V., Sykorova, M., Havranek, E., Determination of Fe, $\mathrm{Zn}, \mathrm{Pb}, \mathrm{Cd}$ and $\mathrm{Se}$ content in medical plants by Xray fluoresence analysis and glavanostatic stripping chronopotentiometric analysis. Acta Facult Univ Comenianae, 55:219-229, 2008. 
22. Sansoni, B., Pandey, V., Analytical techniques for heavy metals in biological fluids. Elsevier, Amsterdam, 91 - 131, 1983.

23. Zutphen, B., Education and training for the care and use of laboratory animals: an overview of current practices. ILAR J, 48:72 - 74, 2007.

24. Renman, R., Jagner, D., Asymmetric distribution of results in calibration curve and standard addition evaluation. Anal Chim Acta, 357:157 - 166, 1997.
25. Plankova, A., Mikus, P., Havranek, E., A clinical study of the relationship of selenium plasma level to atopic dermatitis: a comparison of the results of determination using the methods of galvanostatic dissolution chronopotentiometry and atomic absorption spectrometry. Czech Slov Pharm, 58:109 $-115,2009$.

26. Miller, J., Miller, J., Statistics and chemometrics for analytical chemistry, $5^{\text {th }}$ ed., Pearson Education, Harlow, England, 2005. 